\title{
AUTOMATED CALCULATION OF DIII-D NEUTRAL BEAM AVAILABILITY
}

\author{
by \\ J.C. PHILLIPS, R.M. HONG, and B.G. SCOVILLE
}

This is a preprint of a paper to be presented at the 18th IEEE/NPSS Symposium on Fusion Engineering, October 25-29, 1999, in Albuquerque, New Mexico, and to be published in the Proceedings.

\author{
Work supported by \\ the U.S. Department of Energy \\ under Contract No. DE-AC03-99ER54463
}




\section{DISCLAIMER}

This report was prepared as an account of work sponsored by an agency of the United States Government. Neither the United States Government nor any agency thereof, nor any of their employees, make any warranty, express or implied, or assumes any legal liability or responsibility for the accuracy, completeness, or usefulness of any information, apparatus, product, or process disclosed, or represents that its use would not infringe privately owned rights. Reference herein to any specific commercial product, process, or service by trade name, trademark, manufacturer, or otherwise does not necessarily constitute or imply its endorsement, recommendation, or favoring by the United States Government or any agency thereof. The views and opinions of authors expressed herein do not necessarily state or reflect those of the United States Government or any agency thereof. 


\section{DISCLAIMER}

Portions of this document may be illegible in electronic image products. Images are produced from the best available original document. 


\title{
Automated Calculation of DIII-D Neutral Beam Availability
}

\author{
J.C. Phillips, R.M. Hong, and B.G. Scoville \\ General Atomics, P.O. Box 85608, San Diego, California 92186-5608
}

\begin{abstract}
The neutral beam systems for the DIII-D tokamak are an extremely reliable source of auxiliary plasma heating, capable of supplying up to $20 \mathrm{MW}$ of injected power, from eight separate beam sources into each tokamak discharge. The high availability of these systems for tokamak operations is sustained by careful monitoring of performance and following up on failures. One of the metrics for this performance is the requested injected power profile as compared to the power profile delivered for a particular pulse. Calculating this was a relatively straightforward task, however innovations such as the ability to modulate the beams and more recently the ability to substitute an idle beam for one which has failed during a plasma discharge, have made the task very complex. For example, with this latest advance it is possible for one or more beams to have failed, yet the delivered power profile may appear perfect. Availability used to be manually calculated. This paper presents the methods and algorithms used to produce a system which performs the calculations based on information concerning the neutral beam and plasma current waveforms, along with post-discharge information from the Plasma Control System, which has the ability to issue commands for beams in real time. Plots representing both the requested and actual power profiles, along with statistics, are automatically displayed and updated each shot, on a webbased interface viewable both at DIII-D and by our remote collaborators using no-cost software.
\end{abstract}

\section{INTRODUCTION}

Since the first short pulse neutral beams were made operational on Doublet III, (the predecessor device to DIII-D) availability records have been kept which detail service status for each ion source and a measure of performance for each experimental plasma discharge. If the beam is not in service, or if performance is degraded, the various causes are tallied so that resources may be directed towards the elimination of problems which most directly effect operations. Causes include: power supply, computer, vacuum, cooling water, cryogenic, or other service failures, human error, as well as ion source failures. Historically at DIII-D the highest frequency causes for neutral beams failure are power supply related, followed by computers, cryogen, and other ancillary services. The situation is in a sense self-regulating, if one cause assumes higher than normal visibility, efforts result which tend to restore the status quo. Staff efforts to keep these systems available and running are taken very seriously, and availability has historically been high - greater than $90 \%$ and often exceeding $95 \%$.

The first guidelines for determining availability were somewhat loose. For systems operational but not currently being requested or utilized, the rule was formulated thus: "if we believe a system would run were it requested, then it's deemed available." Of course, as anyone who works with large complicated systems knows, even a system previously operational, once idled, may prove non-functional in need of repair when next summoned to operate.

In prior years, this tally was kept manually and somewhat subjectively. The rule statement was "if a source fulfills $80 \%$ or better of its programmed power profile, score one, if not score zero." This threshold approach was adopted as it was deemed impractical for the beam coordinator to calculate actual percentage fulfillment for each source on each discharge, given other responsibilities. Over successive years the metrics for neutral beam availability gradually became more stringent, so that today's availability rating of $90 \%$ implies better performance than the same figure of 10 years ago. The detailed performance of each ion source, beyond simply being available, was tracked to characterize whether the power profile provided matched that requested for the plasma discharge.

\section{Challenges}

The selected approach simply involves objective comparison of the requested versus actual performance for each source, on a per plasma discharge basis. This is simply stated, but actual application proved to be far from simple. New capabilities developed these past few years using DIII-D's neutral beams have brought benefit to the research program, but at the same time present significant challenge to the task of quickly and reliably determining whether beam pulses for the most recent discharge represent a faithful execution of the request.

These relatively new capabilities include:

Pre-Programmed Modulation: DIII-D's neutral beams may be modulated (or gated in square wave fashion) by several different means. The basic form of modulation can involve more than 200 discrete cycles, pre-programmed for each source. Modulation sequences may also be out of phase with a companion sequence for another ion source (Fig. 1). This gives the ability to finely tune the injected neutral beam power profile, and also the important capability of providing multiple on-off transitions for various DIII-D diagnostics, including charge exchange recombination and beam emission spectroscopy.

Modulation by the Plasma Control System: The plasma control system computer (PCS) extends the functionality of basic modulation giving real-time capability. Neutral beam modulation by the PCS supports real-time feedback control of given plasma parameter, such as density. This real-time modulation causes its own special set of problems because by its nature, the "program" is not known in advance of plasma 


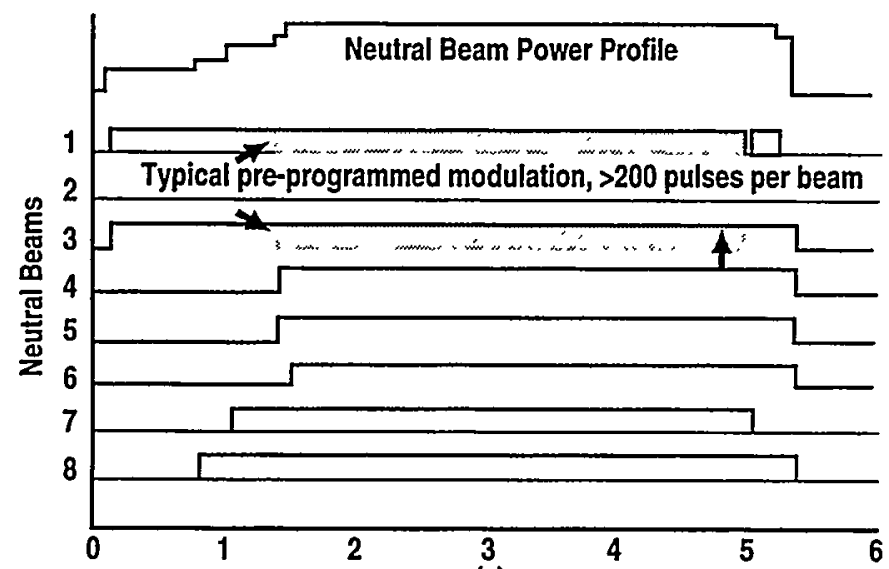

(s)

Fig. 1. Basic pre-programmed neutral beam modulation.

discharge (Fig. 2). Thus, two apparently identical requests on two successive plasma discharges might on the one hand make use a particular beam, and on the other hand not pulse it at all, dependent on the behavior of the controlled plasma parameter. This complicates the definition of required performance to be used in comparison to the actual performance, requiring access to a record of commands issued "on the fly" by the PCS computer during the plasma discharge. Obtaining, yet alone assimilating, this information can be a daunting task for the beam coordinator, given a schedule with as few as $8 \mathrm{~min}$ between discharges.

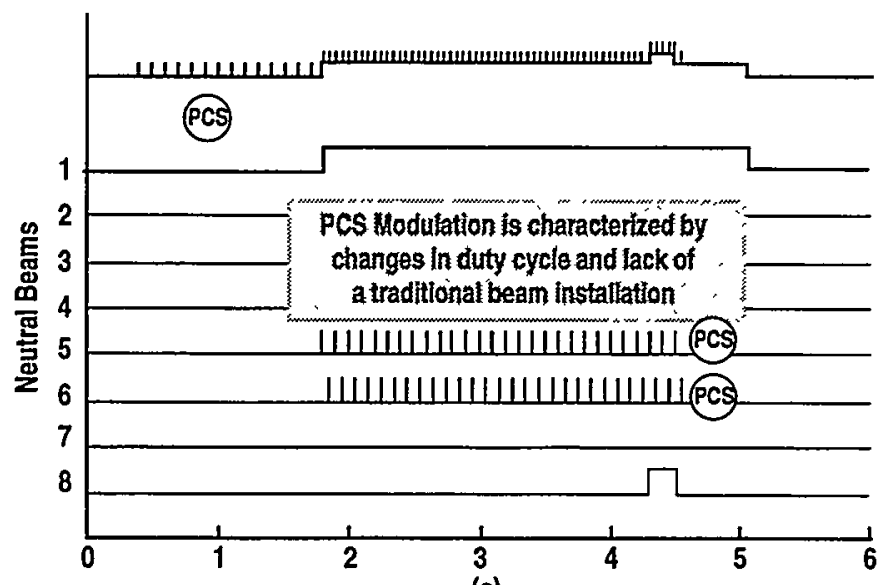

(s)

Fig. 2. PCS real-time modulation adds an unpredictable element.

Neutral Beam Substitution: Most recently, the ability to modulate neutral beams for real-time feedback and control has been extended, allowing the PCS to substitute an idling neutral beam for one requested but failing to perform during the plasma discharge [1]. This scheme works well and is credited with substantially increasing the overall production of many DIII-D discharges (Fig. 3). Providing any assessment of this performance is problematic, to say the least. For example, if six of an inventory of eight ion sources are requested to

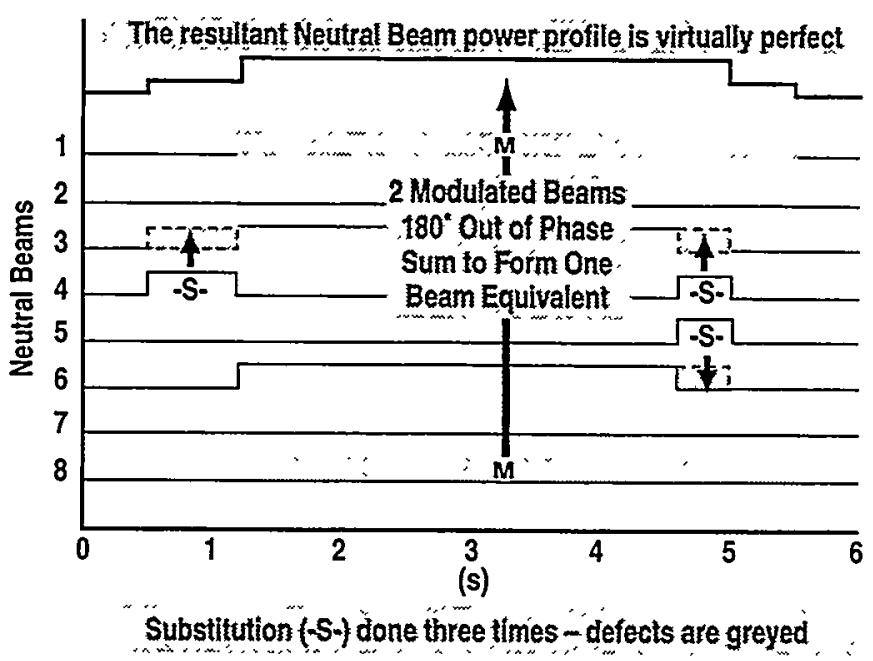

Fig. 3. Substitution in real-time of one neutral beam for another in case of interrupts or failure adds another layer of complexity.

perform for a particular plasma discharge, and one fails but is substituted for by one of the two idle sources, the apparent availability is $100 \%$. However treated as such, the ability to track failures is lost.

Neutral Beam Timing Issues: To permit these enhanced modulation modes and beam substitution, neutral beam staff have been required to make significant changes to the basic systems for the timing of a beam pulse, and these changes have also impacted availability calculations. For example, for a particular neutral beam to be slated as "available for substitution" on behalf of any number of other beams participating in the plasma discharge, it must have what appears to be a very long pulse length enabled, perhaps as long as seven to nine seconds. Of course, to be ready on a few milliseconds notice for substitution, the source filaments and arc discharge are required to have been established as normal and for time periods in excess of those previously considered normal. The most serious implication, is that if a neutral beam were to inadvertently fire for a substantial portion of this long standby period, significant and costly damage could be done to the beamline internals. To mitigate this serious problem, a real-time interlock has been developed [2] with redundant safety features, which act to limit the accumulated beam ontime for a given source on a specific discharge to safe limits, given knowledge of the power levels involved.

\section{IMPLEMENTATION}

This system for availability accounting grew incrementally over the 1999 operating campaign. Design was made difficult by the disparate sources for the requisite information for the calculations. These data include a) pre-programmed timing entered by the timing operator $b$ ) traces representing neutral beam voltages for each source $c$ ) substitution parameters from the timing operator d) position of various hardware controls and interlocks to do with the source of timing information e) substitution commands or feedback control commands, if any, issued by the PCS computer. 
The processing required to produce the display and its content is distributed across two (a) and (b) computer systems (Fig. 4), a) one of the two beam control computers running the REALIX operating system, Unix with real-time extensions, b) a PC using the Linux operating system which acts as a dedicated web server for the neutral beam group. Client-server socket-based messaging summons data from other control and acquisition computers, and similar messaging retrieves time dependent shot based data from DIII-D's PTData data archive. Some processing is done on the beam control computer including generating the plots using the Unix GNUPLOT package. These plot files are then run through another filter converting them to GIF files suitable for Web display, and through a filter, written by the author to substitute the color map in the GIF files with a standard scheme. These data files are passed to the Linux machine by means of disk space NSF mounted to the two beam control computers. The Linux machine then tends to further detail and serves it up, - to itself - since the Linux machine also provides the computing power to run the $\mathrm{X}$ Window flavored Netscape clients, as well as running the Apache Web Server. This combination provides the content and manages the server-push/auto-refresh of every subscribed client. This highly unusual (some may term incestuous) relationship, works very well in practice, ensuring that the beam group does not add the burden of additional web browsers to DIII-D's scientific computing machines.
To provide an easy to use, helpful display one important design criteria called for automatic refresh, or hands-free operation. Consistent with the beam group's desire to utilize no or low cost client-side tools, and to make our data available beyond the reach of the control room operator's proprietary and licensed display software, a web browser interface was chosen, employing "server-push" for refresh.

The motivation for requiring that the display automatically updates, is that working in the DIII-D control room, the minutes leading up to and just after a plasma discharge can be demanding. If a discharge does not go as well, there is also good likelihood the beam coordinator will soon be face to face with more than one person requesting information as to what happened. Given the tools of just a year ago and the advent of the substitution and modulation advances described, an answer to this question was becoming increasingly difficult to obtain quickly, if at all. This work (Fig. 5) seeks to make the Beam Coordinator position tenable by supplying accurate information in a timely manner.

The user interface shows simply the requested beam power profile, the "as ran" power profile, the accumulated ontime and status for each ion source. In addition an availability score is calculated for each ion source. Given the overall reliability of our systems, more often than not these figures are $100 \%$.

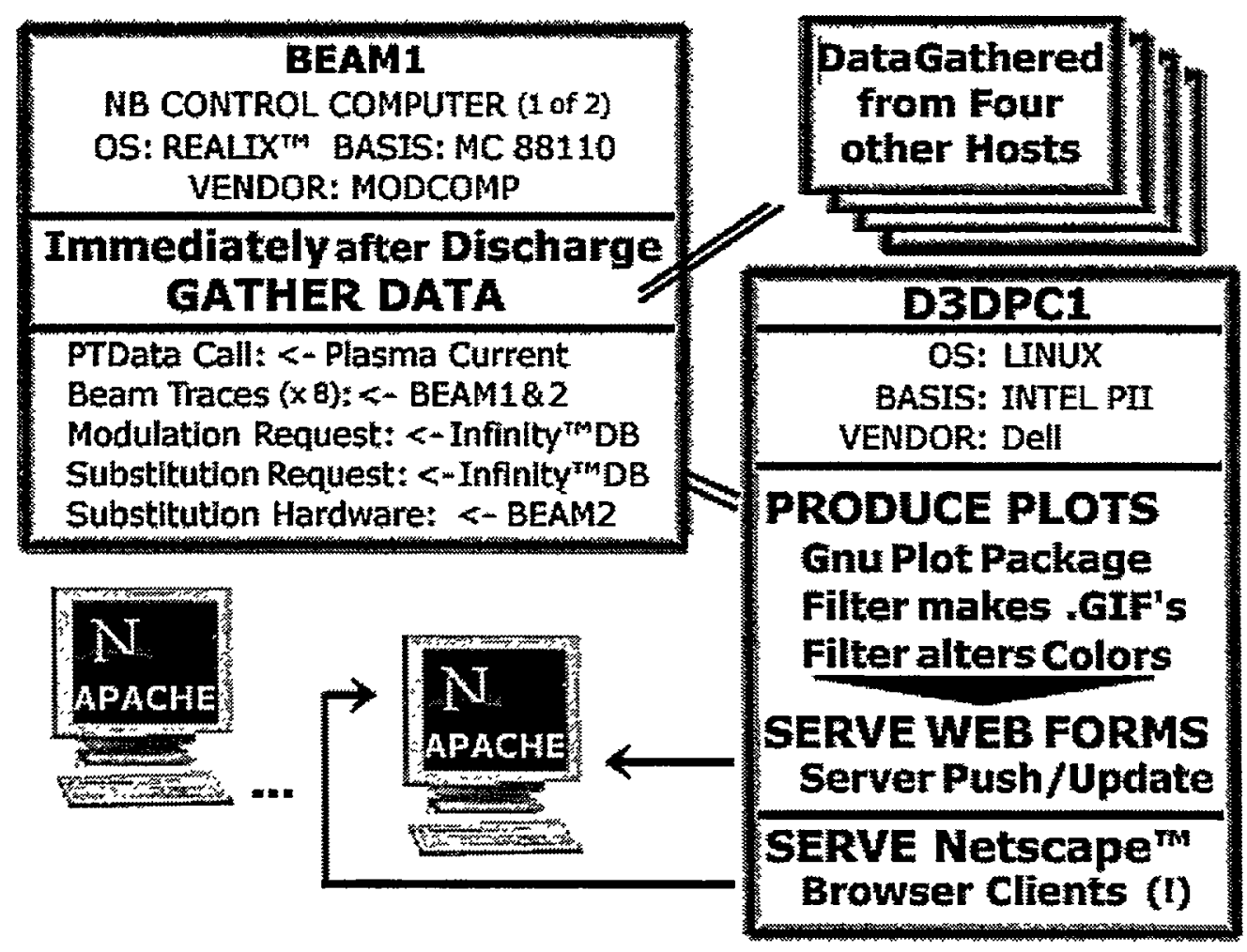

Fig. 4. Processing is distributed amongst several systems. 


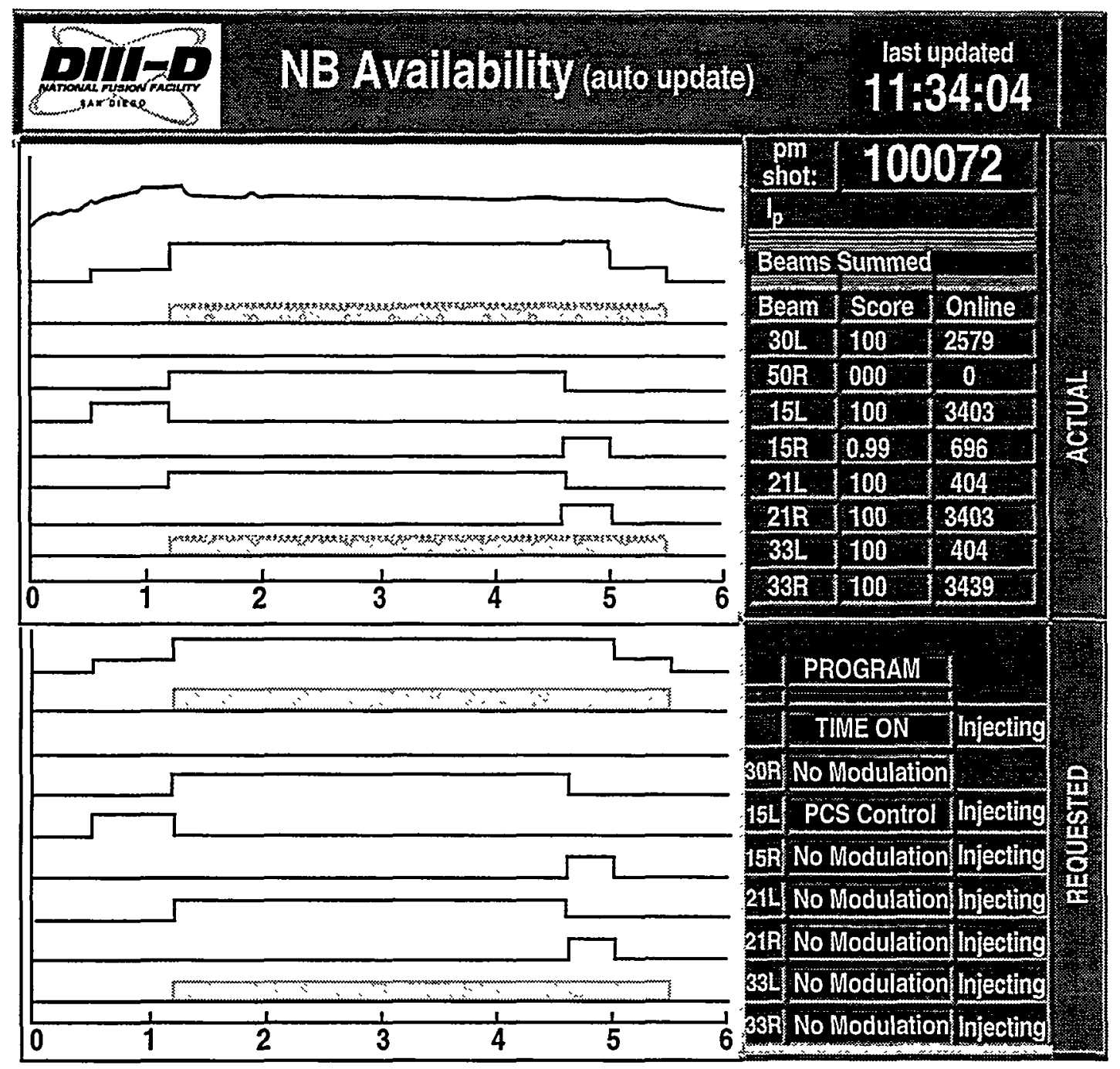

Fig. 5. The web-based user interface for display of neutral beam availability.

\section{CONCLUSION}

While presenting various challenges during implementation and testing, the neutral beam availability software now faithfully executes for each plasma discharge, day in day out. As a benefit of the methods chosen, multiple iterations of the World Wide Web based display may be run concurrently, meaning that anyone with an interest and password may view the auto-refreshing display, whether located in their remote office, at home, or in the DIII-D control room. The simple fact that the display has become a commonly used management tool is validation sufficient for the authors that the project was worthwhile. Just after a plasma discharge, should the display indicate neutral beam's performance may have faltered, the neutral beam operations crew in the control room often simply needs mark the seconds until the telephone rings inquiring whether we ourselves are in need of added support or assistance, to provide reliable service to the DIII-D team.

\section{ACKNOWLEDGMENT}

Work supported by U.S. Department of Energy under Contract No. DE-AC03-99ER54463. The authors gratefully acknowledges the encouragement, support, and acceptance of DIII-D's computer group for the implementation of these methods, some of which break fresh ground and some of which are experimental and bound for improvement.

\section{REFERENCES}

[1] J.C. Phillips, et al., "An Algorithm to Provide Real Time Neutral Beam Substitution in the DIII-D Tokamak," Proc. 11th IEEE/NPSS Real Time Conf., Santa Fe, New Mexico, June 1999.

[2] D.S. Baggest, et al., "Extending DIII-D Neutral Beam Modulated Operations with a CAMAC Based Total on Time Interlock," these Proceedings. 